\title{
El subsidio a la demanda educativa en la asistencia a la Educación Media en Uruguay
}

Education subsidy to attendance at Secondary (Middle) Education in Uruguay

Subsídio à demanda educacional na assistência ao Ensino Médio no Uruguai

\section{DOI: https://doi.org/10.18861/cied.2021.12.1.3064}

Pablo Menese Camargo

Centro de Estudio de Políticas Educativas, UdelaR

Uruguay

nese@gmail.com

ORCID: 0000-0001-5940-8703

Recibido: 05/05/2020

Aprobado: 15/08/2020

Cómo citar:

Menese Camargo, P. (2021). El subsidio a la demanda educativa en la asistencia a la Educación Media en Uruguay.

Cuadernos de Investigación Educativa, 12(1). https://doi.org/10.18861/cied.2021.12.1.3064

\section{Resumen}

En Uruguay las tasas de egreso de Educación Media son cercanas al 40\%, de las más bajas de toda América Latina. Una de las estrategias de combate a la desigualdad educativa fue, en el año 2008, la ampliación, el refuerzo y la focalización de las Asignaciones Familiares, uno de los programas de transferencia monetaria condicionada más antiguos y de mayor alcance. De este modo, a traves del incremento de una partida monetaria mensual se subsidia el gasto educativo privado. y los individuos provenientes de los hogares de menores ingresos pueden afrontar los costos de continuar escolarizados. invirtiendo en capital humano y rompiendo el ciclo de la vulnerabilidad económica. El objetivo de este trabajo es observar el efecto de un programa de subsidio al gasto educativo privado en la asistencia a la Educación Media. Para ello se utiliza una serie armonizada de la Encuesta Continua de Hogares desde el año 2001 al 2017 en la cual, a través de una combinación de cuasi experimentos tales como Propensity Score Matching y Difference in Differences se evalua el impacto de las Asignaciones Familiares y de la ampliacion de regimen desde el año 2008, en la asistencia a la Educacion Media Basica Educacion Media Superior. Entre los principales resultados se destaca el efecto significativo y positivo de las Asignaciones Familiares en la asistencia a la Educacion Media Basica, con un incremento de la magnitud luego de la reforma de 2008 En cambio, en Educación Media Superior las Asignaciones Familiares tuvieron un efecto nulo previo a 2008, y negativo posteriormente.

Palabras clave: capital humano - transferencias condicionadas - desigualdad educativa.

\section{Abstract}

In Uruguay, Secondary or Middle School graduation rates are close to 40\%, recording one of the lowest rates throughout Latin American countries. One of the most important strategies to fight against educational Inequality was implemented in 2008, pursuing expansion and one of the oldest and most extensive conditional cash transfer programs. The purpose of this subsidy is to support educational expenses to families through a monthly monetary allowance, so that individuals from lower income households can face the costs of schooling, investingin human capital so as to break the cycle of economic vunerability The objective of this work is to observe the effect of the subsidy program on private educational expenditure regarding attendance at Secondary School. For this propose, using a harmonized series of the Continuous Household Survey from 2001 to 2017, two quasi-experiments techniques are combined. Propensity Score Matching and Difference in Differences. In this way, the impact of expansion implemented through Asignaciones Among the main results, the allowance of A signaciones Familiars evidenced a significant and positive effect in the attendance at Lowersecondary School with great increase after the reform of 2008. However, in Upper Secondary Education. Asignaciones Familiares subsidy had no effect prior to 2008, and thereafter was negative.

Key words: Human capital - conditional transfers - educational inequality.

\section{Resumo}

No Uruguai, a taxa de egresso do Ensino Médio está perto de 40\%, das mais baixas da América Latina. Uma das estratégias de combate à desigualdade educacional foi, no ano 2008, a amplificação, o reforço e a focallzaçao das Asignaciones Familiares (auxillio ds de maior abrangência Assim através de um aumento mensal da transferência monetária as despesas educacionais particulares são subsidiadas, aos fins de que os individuos de familias de baixa renda possam arcar com os custos de continuar a escola, investindo em capital humano e quebrando o ciclo da vulnerabilidade econômica. O objetivo desse trabalho é observar o efeito de um programa de subsidio de despesas educacionais particulares na assistência ao Ensino Médio. Para tanto, é utilizada uma série harmonizada de a Pesquisa Domiciliar Continua de 2001 a 2017, na que, atraves de uma combinação de quase experimentos como Propensity score Matching e Difference in Differences, o impacto das Asignaciones Familiares e avatiado, alem da expansão do regime a partir de 2008, na assistência ao Ensino Medio Basico e ao Ensino Medio Superior. Dentre os na assistência ao Ensino Médio Básico com um aumento importante após a reforma de 2008 Porém no Ensino Médio Superior as Asignaciones Familiares têm um efeito nulo antes de 2008, e negativo posteriormente.

Palavras chave: Capital Humano - Transferencias Condicionadas - Desigualdade Educacional 


\section{Marco teórico y antecedentes}

\section{El subsidio al gasto educativo}

Un individuo que desea acumular años de estudio necesita invertir, lo que hace a un gasto que se expresa de dos maneras: gasto público y gasto privado. El gasto educativo público son los costos que asume un colectivo para contribuir a que un grupo de individuos acceda a determinado nivel educativo (Woodhall, 2004). A lo largo de la historia el costo educativo público ha recaido en diferentes colectivos, y ha priorizado diferentes niveles educativos. Desde finales del siglo XIX en Uruguay, el Estado ha venido asumiendo los costos educativos públicos, priorizando elacceso universala la educación primaria como nivel educativo formal obligatorio. Estos primeros costos implicaban la construcción y mantenimiento de centros educativos en todo el territorio nacional, el equipamiento de materiales de estudio básicos, la formación de profesionales encargados de dictar clases en tantos centros como hubiere, los salarios docentes y no docentes y otros costos relativos a todo el funcionamiento del sistema en general.

El segundo tipo de gasto educativo, el gasto educativo privado, es aquel que asumen los hogares para que sus integrantes se escolaricen una cantidad determinada de años. La forma del gasto privado se expresa como: i) costo directo como el de transporte, materiales de estudio, uniformes y alimentos, entre otros; y ii) costo indirecto, como la renuncia a un salario dentro del mercado laboral, por estar inserto en el sistema educativo y no en el mercado laboral (Woodhall, 2004). El gasto educativo privado, que generalmente queda librado a los hogares, es relativo a varios elementos, entre ellos el ciclo educativo que cada integrante cursa, la cantidad de integrantes escolarizados en el hogar, los ingresos totales del hogar y la edad de cada individuo escolarizado. Este último elemento -la edades de particular importancia ya que incrementa los costos directos, por ejemplo, en el consumo de alimentos y ocio, pero también los costos indirectos. La edad mínima legal para trabajar son los 14 años, y de ahí en más la tasa de empleo y el salario potencial se incrementan como una función de la edad.

Se entiende entonces que el hogar de origen y, en definitiva, el costo relativo al gasto educativo privado de cada integrante escolarizado en el hogar, son una importante fuente de desigualdad educativa, máxime considerando a la escolarización como una inversión (Becker, 1993), importante tanto para el bienestar de los individuos como para la reproducción y evolución de la sociedad en general (da Silveira, 2009; Sen, 2000). Por consiguiente, el Estado decide incrementar su gasto educativo público contribuyendo al subsidio del gasto educativo privado a través de programas de transferencias (monetarias y en especie) a los hogares, cada vez que haya alguien escolarizado en dicho hogar. Este tipo de programas no es universal, y esto ocurre en virtud de que el gasto educativo privado está relacionado -entre otros factores- a los ingresos del hogar. Por ende, hay hogares donde los ingresos per cápita superan ampliamente los costos directos e indirectos, mientras que hay hogares donde los ingresos per cápita no superan ningún costo. Es para este último tipo de hogares que se diseñan y donde se aplican los programas de subsidio al gasto educativo, destinados a reducir la desigualdad educativa asociada al hogar de origen que implica la reproducción intergeneracional de la escolarización, incrementando el espiral de la desigualdad manifiesta en la estructura social. 


\section{Las Asignaciones Familiares}

El programa de Asignaciones Familiares se creó en el año 1943 por la Ley 10.449, y fue una parte importante de la temprana matriz de bienestar nacional que buscaba complementar el salario de los trabajadores formales con hijos de hasta 17 años bajo ciertas condiciones, entre ellas, la asistencia a la educación formal (Midaglia, 2009). Originalmente era un programa de tipo universal-corporativo, aunque desde 1995 la prestación se destinaba a los trabajadores de menores ingresos, definidos como 10 salarios mínimos nacionales. Posteriormente, en 1999, y a través de la Ley 17.139 se incluyen dentro de los beneficiarios a los hogares en los que la mujer es el único sustento del hogar, a los trabajadores hombres y mujeres una vez finalizado el seguro de desempleo, y a mujeres embarazadas y hasta a un año del nacimiento, en todos los casos con ingresos menores a 3 salarios mínimos nacionales.

En el año 2004, luego del saldo negativo en términos de bienestar social producto de la crisis del 2002, la Ley 17.758 extiende el beneficio de las Asignaciones Familiares a todos los hogares con ingresos mensuales de hasta 3 salarios minimos nacionales, levantando definitivamente la condición de la aportación a la seguridad social (Amarante, Arim, de Melo \& Vigorito, 2009). Hacia finales del 2004 se sustituye la medida que indexa las transferencias desde el Salario Mínimo Nacional hacia la de Base de Prestaciones y Contribuciones, la cual se ajusta en función de la variación del Índice de Precios al Consumo (Bucheli, Cabella \& Vigorito, 2005). Esto representó un avance sustantivo en términos de equidad y de eficiencia del programa, en virtud de superar los problemas de indexar por el Salario Mínimo Nacional, en un marco de depreciación del salario real que se había observado durante toda la década de 1990 y principios de la década del 2000. En el año 2008, a través de la Ley 18.227 el programa de Asignaciones Familiares se divide, y una parte se une al Plan de Equidad bajo la órbita del MIDES (Ministerio de Desarrollo Social). De este modo ingresa en las políticas y reformas iniciadas desde el año 2005 en el paquete de políticas sociales propuesto y ejecutado por el primer gobierno de izquierda. Desde entonces existen dos tipos de Asignación Familiar: Las Asignaciones Familiares de tipo contributivo aún bajo la órbita del BPS (Banco de Previsión Social), que representan las transferencias del viejo modelo previo a la reforma del 2004, y las Asignaciones Familiares no contributivas del Plan de Equidad bajo la órbita del MIDES (DINEM-MIDES, 2015).

Sobre las Asignaciones Familiares contributivas, son transferencias mensuales destinadas a los niños y jóvenes de o a 17 años, administradas por la madre/ padre/tutor, siempre que en el hogar haya al menos un trabajador formal, jubilado o pensionista del BPS, con un ingreso total del hogar de hasta $\$ 47.491\left(1 .^{\circ}\right.$ de enero de 2018). Los montos de la transferencia varian de acuerdo a la cantidad de beneficiariosya los ingresos del hogar, siendo de $\$ 756$ por beneficiario con ingresos del hogar de hasta $\$ 28.489$ (1..$^{\circ}$ de enero de 2018), y de $\$ 378$ por beneficiario con ingresos del hogar entre $\$ 28.489$ y $\$ 47.491\left(1 .^{\circ}\right.$ de enero de 2018). Con respecto al sistema no contributivo, las Asignaciones Familiares del Plan de Equidad incluyen una serie de disposiciones que lo diferencian significativamente del esquema anterior. Entre ellas se encuentran: i) cambio en el algoritmo de selección de beneficiarios, adoptando el Índice de Carencias Críticas elaborado por el Instituto de Economía de la Facultad de Ciencias Económicas y de Administración de la Universidad de la Republica, el cual clasifica los hogares en un indice de 0 a 1 de acuerdo con su nivel de vulnerabilidad en términos sociales, económicos y materiales (DINEM-MIDES, 2014; Vigorito, Arim \& Amarante, 2005); ii) incremento en el valor de la trasferencia.

Las contraprestaciones para ser beneficiario de uno y otro programa son muy similares. En el caso de las Asignaciones Familiares no contributivas del Plan de 
Equidad especificamente, se solicita contar con cedula de identidad a partir de los 45 dias del nacimiento del niño, y controles médicos para los menores de 4 años. Los demás elementos son comunes a ambos sistemas de Asignaciones. En cuanto a la salud, son los controles periódicos a embarazadas, y con respecto a lo educativo, educación primaria a partir de los 4 años y hasta los 14 años, educación media de 14 a 18 años, y para el caso específico de las Asignaciones Familiares contributivas existe una condición más por la cual todo aquel que a los 16 años no haya culminado su educación primaria debe justificar esta situación extraordinaria (DINEM-MIDES, 2015).

El programa de Asignaciones Familiares ha sido diseñado como una transferencia condicionada a la asistencia a la educación formal obligatoria por parte de los integrantes del hogar en edad de escolarizarse. En caso de no cumplir con tal requisito, la transferencia es suspendida hasta que la asistencia al sistema educativo sea retomada. La transferencia ocurre siempre que exista un beneficiario potencial en el hogar, no existiendo necesidad de renovación y estando sujeta -en el caso de las transferencias no contributivas- a visitas del equipo MIDES. Por tanto, el diseño de las Asignaciones Familiares como subsidio a la demanda educativa en Educación Media debiera generar incentivos a la permanencia educativa, y no asi a la acreditación ni de año ni de nivel ya que la misma no está condicionada a resultados sino a la asistencia. Esta consideración es central a la hora de pensar la evaluación del programa, ya que define la naturaleza del efecto esperado.

Ambos programas de Asignaciones Familiares han acumulado un conjunto significativo de evidencia empírica sobre el efecto de la trasferencia en un amplio abanico de dimensiones, tales como el combate a la pobreza, la redistribución del ingreso y, muy particularmente, la asistencia a la educación formal obligatoria. Probablemente el principal antecedente por su exhaustividad y convergencia temática con el presente trabajo sea el estudio llevado adelante por Machado et al. El nuevo régimen de Asignaciones Familiares. Su impacto en la participación y permanencia en el Ciclo Básico de Educación Media (Machado et al., 2012). Por medio de la utilización de diferentes microdatos tales como los registros administrativos del sistema electrónico de la ANEP SECLI (Administración Nacional de Educación Pública - Secretarías Liceales), los registros administrativos de UTU (Universidad del Trabajo del Uruguay) y BPS (Banco de Previsión Social) y PIU (Programa Liceos con Tutorias) del 2009 evalúan: i) el impacto de las Asignaciones Familiares del Plan de Emergencia en la asistencia al sistema educativo formal obligatorio, y; ii) el cambio del sistema de Asignaciones Familiares en 2008.

Por medio de un diseño cuasi experimental del tipo Propensity Score Matching, se parea a la población por sexo, edad e Índice de Carencias Criticas, y se observan asistencia y promoción para diferentes subgrupos de beneficiarios. Se encuentra un efecto significativo con respecto al impacto de las Asignaciones Familiares del Plan de Equidad en la asistencia de un 11\% entre los jóvenes de 12 a 17 años, que al desagregarla entre los de 12 a 14 años es de un 9\%, mientras que entre los de 15 a 17 años es de un 18\%. No obstante, este efecto es un efecto edad y no un efecto nivel educativo.

Un aporte sustantivo del trabajo es la estimación del peso relativo del impacto de la Asignación Familiar en relación al monto de la transferencia, donde para quienes reciben transferencias con un valor por debajo de la mediana la asistencia baja al 5\%, mientras que para quienes reciben transferencias con un valor por encima de la mediana, la asistencia asciende a un $12 \%$. Con respecto al cambio del modelo de Asignaciones Familiares se observa un efecto significativo, aunque existe un problema en el cambio de la composición de la población ya que al cambiar y ampliar el criterio de selección de la población beneficiaria cambia la composición del grupo de tratamiento y control, lo que genera alteraciones en las estimaciones. 
Por último, no se encuentran efectos significativos respecto a las transferencias ni a la acreditación, así como tampoco en el descenso de insertos en el mercado laboral.

Respecto al estudio en general se desea destacar lo siguiente: i) la transferencia no posee un efecto homogéneo en la población vulnerable, mientras que parece ser un efecto relativo -entre otros aspectos- a los ingresos del hogar de origen; ii) no hay un descenso en quienes están insertos en el mercado laboral, por lo tanto el subsidio al gasto educativo del hogar no modifica el trade off entre trabajo y estudio, y: iii) la transferencia genera un efecto significativo en la asistencia pero no en la acreditación, por tanto, parece que el subsidio al gasto educativo contribuye a que los jóvenes asistan al sistema educativo, pero no a que acrediten los niveles. Con respecto al cambio del sistema de Asignaciones Familiares en 2008, es pertinente revisar el trabajo de Amarante etal. Transferencias de ingresosy asistencia escolar. Una evaluación ex-ante de esquemas alternativos en Uruguay (Amarante et al., 2009). En este caso, utilizando como fuente de datos la ECH (Encuesta Continua de Hogares) del 2006, especificamente adolescentes de entre 14 y 17 años, estiman a través de un modelo lineal generalizado de tipo multinomial la probabilidad de que un joven escoja entre estas opciones: i) estudiar, ii) trabajar, o iii) trabajar y estudiar. Para valorar esto se toma en cuenta el hogar, estimando el ingreso ficto del joven y sumándolo al del hogar.

El resultado principal es que un aumento en el monto de la transferencia -parte del cambio del sistema de Asignaciones Familiares- incrementa la asistencia a la Educación Media Básica de los jóvenes de manera progresiva, ya que es una función relativa al hogar de origen. Es decir, al incrementar la transferencia, la asistencia al Ciclo Básico se incrementa, en particular para aquellos hogares donde el valor relativo de la transferencia respecto a los ingresos totales del hogar es mayor. Dos elementos a destacar son: en primer lugar, que no se ven efectos significativos en el cambio de sistema para la educación media superior y, en segundo lugar, la estimación se realiza con los ingresos fictos efectivos pero no se estiman posibles ingresos futuros, lo cual parece un elemento importante a la hora de decidir si continuar invirtiendo en capital humano o no.

Una vez concretado el cambio de régimen de Asignación Familiar han surgido evaluaciones especificas del programa asociado al Plan de Equidad y de la eficiencia de la cobertura e impacto del mismo. El trabajo Evaluación impacto del programa Asignaciones Familiares-Plan de Equidad y la Tarjeta Alimentaria del MIDES (Bérgolo, Dean, Perazzo \& Vigorito, 2015) utiliza microdatos de la encuesta de seguimiento a los beneficiarios de las Asignaciones Familiares del Plan de Equidad junto con registros administrativos de ANEP (sistema SECLI) y UTU para estimar el impacto del programa de transferencias en, por ejemplo, la asistencia a la educación formal obligatoria. La metodología es de tipo cuasi experimental, especificamente Regresión Discontinua, y utiliza el Índice de Carencias Críticas normalizado en el eje de las abscisas y la proporción de la asistencia a la educación media en el eje de las ordenadas. Los dos principales resultados apuntan a que: i) existe un efecto significativo de entre el $2 \%$ y el $4 \%$ en la asistencia a la educación media para el grupo etario de entre 13 y 17 años, y; ii) al desagregar las edades se observa que el efecto significativo disminuye para cada año hasta dejar de ser significativo a los 17 años. Por tanto, parecería que el efecto de la transferencia no solo es relativo a los ingresos del hogar de origen sino también a la edad.

Con respecto a los informes especificos de organismos estatales, hay uno en concreto que es pertinente a efectos del trabajo, incluso sin evaluar la asistencia a la educación formal obligatoria. Análisis y perspectivas para los programas de transferencias: Asignaciones Familiares y Tarjeta Uruguay Social (DINEM-MIDES, 2015) realiza un análisis de incidencia de microsimulaciones aritméticas no comportamentales estáticas. Por medio del uso de la ECH 2013 se simulan diversos 
escenarios con el objetivo de analizar la equidad redistributiva que generan las Asignaciones Familiares y la Tarjeta Uruguay Social. Se propone una simulación de la expansión de las Asignaciones Familiares a jóvenes de entre 18 y 21 años, lo cual fue parte del debate político en 2014, y se propone eliminar la condición de la asistencia a la educación formal obligatoria en virtud de que durante este periodo se acumulan los eventos de transición a la adultez, por tanto, concluyen que ya se inició el pasaje del sistema educativo al trabajo.

El informe muestra asistencia o acreditación frente a la inasistencia o no acreditación de la educación media básica por decil de ingreso entre los jóvenes de entre 18 y 21 años que, sin ser titulares, pertenecen a hogares beneficiarios de Asignaciones Familiares del Plan de Equidad. Se observa que, para el primer decil, la asistencia o acreditación de la enseñanza media básica supone menos del 20\%, mientras que la no asistencia o no acreditación representa cerca de un 35\%. Es en este mismo decil donde se encuentra la mayor proporción de hogares donde los menores no asisten a la educación formal obligatoria. Del decil 2 en adelante la tendencia se revierte, predominando quienes asisten o acreditaron, frente a quienes no asisten o no acreditaron, por una diferencia de entre 3 y 5 puntos.

En términos generales este resultado es consistente con el capitulo Uruguay: evaluación de las politicas de transferencias (Vigorito \& Colafranceschi, 2013), donde se observa una comparación en una serie de dimensiones entre el 2006 y el 2011, en el que los percentiles de menores ingresos han incrementado globalmente su escolarización promedio. En cualquier caso, los dos elementos a destacar son que la presencia de la transferencia parece ser una externalidad positiva en la asistencia o acreditación en la educación media básica incluso para los no titulares del programa y que, probablemente, los eventos de transición, que en general ocurren antes de los 18 años, configuran un decil 1 con un importante rezago en la asistencia a la educación media.

Por último, si bien las condiciones de la contraprestación parecen claras, y más allá de la evaluación del impacto del programa de transferencias en sí, parece razonable reseñar el trabajo Programas de transferencias, condicionalidades y derechos de la infancia (Rossel, Courtoisie \& Marsiglia, 2014), en el cual se entrevista a una muestra aleatoria a 14 familias de Montevideo y Canelones, sancionadas en 2013 con la quita de las transferencias por no cumplir con alguna de las contraprestaciones. Entre las conclusiones se destaca, en primer lugar, que no hay claridad sobre las razones por las que les fueron quitadas las transferencias. De hecho, solo el 18\% de los entrevistados sabía que tenía como contraprestación asistir a educación media, frente a un $42 \%$ que sabía de la condicionalidad de asistir a educación primaria. Al ser entrevistados los jóvenes a quienes se les retiró la transferencia, las razones por las que dejaron de asistir al sistema educativo, en general, declaraban desinterés en las clases, incompatibilidad con tareas de cuidados del hogar a hijos u otros dependientes, por trabajo, o para ayudar a la familia, con lo cual, de ser el caso, parecería que el monto de la transferencia no era suficiente en la función de elasticidad entre trabajo/cuidados y estudio.

\section{Hipótesis}

- Hipótesis 1: el subsidio a la demanda educativa genera efectos positivos y estadisticamente significativos en la persistencia en la educación media básica. Esto ocurre en virtud de que el subsidio logra cubrir el costo educativo de continuar estudiando, y que la utilidad dentro de los primeros años de educación media es baja e implica trabajar antes de los 14 años, la edad mínima legal para hacerlo.

- Hipótesis 2: el subsidio a la demanda educativa genera efectos positivos y estadisticamente significativos en la permanencia en la educación media superior. 
Esto ocurre en virtud de que el subsidio logra cubrir el costo educativo de continuar estudiando, y que la utilidad de acreditar la educación media superior es mayor que la de no hacerlo.

\section{Diseño, método y datos}

Se propone un diseño de tipo cuasi-experimental que asegure: i) asignación, donde es necesario especificar el criterio de selección de la población tratada; ii) estimación, donde es preciso realizar buenas y múltiples mediciones prepost tratamiento para identificar sus efectos puros; iii) grupos de comparación, los cuales permiten generar controles contrafactuales, y; iv) estímulo, donde es necesario identificar puntos de corte exactos asociados a la aplicación del estímulo. No obstante -y a diferencia de los diseños experimentales puros- en general las estimaciones tienen limitaciones y, en algún sentido, las conclusiones son relativamente ambiguas (Campbell \& Cook, 1979; Shadish, Cook, \& Campbell, 2002). El método escogido para estimar el impacto de las Asignaciones Familiares en la permanencia en Educación Media es Difference in Differences, que consiste en comprar la diferencia de dos series pre-tratamiento en relación a la diferencia de las mismas dos series post-tratamiento (Imbens \& Wooldridge, 2009).

Con respecto al problema de investigación planteado se observa la diferencia en la asistencia a Educación Media Básica y Media Superior, desde el 2001 y hasta el 2017. considerando como punto de corte pre-post la nueva modalidad de Asignación Familiar del Plan de Equidad introducida a partir del año 2008. Evidentemente, diff in diff es una técnica cuya eficiencia depende de una importante cantidad de decisiones, ancladas en elementos tanto empíricos como teóricos. Una primera consideración corresponde al indicador escogido para la evaluación del efecto, el que en este caso corresponde a la asistencia a la educación formal obligatoria, en primera instancia en Educación Media Básica y Educación Media Superior. La razón de escoger el indicador de asistencia se corresponde con el diseño de la política, en el cual no existe un estímulo por la acreditación de grado o nivel, sino que se renueva o cancela, condicionada a la asistencia a la educación formal. Un segundo elemento del constructo corresponde al momento en el que aplicar el estímulo. Para este ejercicio se utiliza el 2008, año en el cual entra en vigencia la reforma del sistema de Asignaciones Familiares, cuando se implementa una serie significativa de cambios, incrementándose el monto, la cobertura, a la vez que se cambia el criterio y el método de focalización.

La tercera consideración sobre el constructo del diff in diff es lo que concierne al grupo de tratamiento y al grupo de control. Esto implica que la estimación será tan buena como lo sean los grupos de tratamiento y de control debido a que es este elemento el que limpia de efectos ecológicos o sobreestimaciones y subestimaciones, producto de tamaños o sesgos muestrales (Conley \& Taber, 2011; Donald \& Lang, 2007). Para esto se propone utilizar otro diseño de tipo cuasiexperimental, concretamente Propensity Score Matching (PSM), el que permite generar grupos de tratamiento y de control en función de una serie de variables teóricamente relevantes, a la vez que observar el efecto de la aplicación de un estímulo sobre el grupo de tratamiento (Abadie \& Imbens, 2006). La función de emparejamiento del grupo de tratamiento y de control varía en función de la variación del criterio de selección de la población beneficiaria para cada año desde el 2001 hasta el 2017. Existen dos grandes versiones de cómo emparejar la población. La primera, del tipo Average Treatment Effect (ATE), que puede definirse como el efecto promedio que se debería observar si todos los individuos -en el grupo de tratamiento y de control- reciben el estímulo, comparado con aquellos -también de los grupos de tratamiento y control- que no han recibido el estímulo. 
Por su parte, los datos provienen de una serie sistematizada y armonizada de las Encuestas Continuas de Hogares (ECH) 2001-2017. La utilización de este tipo de microdatos tiene una serie de ventajas y desventajas, que determinan el alcance de las inferencias y representan buena parte del trabajo de armonización y sistematización de datos. En resumen, la ECH es la encuesta periódica y pública de mayor alcance, con una muestra de carácter nacional realizada de manera ininterrumpida desde 1968 y por tanto: i) posee validez externa; ii) tiene un gran número de casos, habilitando múltiples aperturas con bajas posibilidades de obtener celdas vacías, y; iii) son los microdatos con los que se construye una gran variedad de estadísticas y series de diversa naturaleza, por tanto hay un consenso sobre su confiabilidad y -salvo cambios en la definición de indicadores que ya han sido armonizados- la confianza se amplía hacia la consistencia de las series al observar diferentes indicadores por años.

Tabla 1. Criterio de asignación ASFAM de acuerdo a la legislación vigente para cada año en Uruguay

\begin{tabular}{|c|c|}
\hline Año & Criterio de selección \\
\hline 2001 & \multirow{4}{*}{$\begin{array}{l}\text { Varios criterios: } \\
\text { i) Hogares contributivos a la seguridad social, con ingresos totales por debajo de } 10 \\
\text { salarios mínimos nacionales. } \\
\text { ii) Hogares no contributivos donde la mujer es el único sustento del hogar, con } \\
\text { ingresos totales por debajo de } 3 \text { Salarios Minimos Nacionales. } \\
\text { iii) Hogares no contributivos donde haya finalizado el seguro de desempleo, con } \\
\text { ingresos totales por debajo de } 3 \text { Salarios Minimos Nacionales. } \\
\text { iv) Mujeres embarazadas no contributivas y con hijos de hasta } 1 \text { año de nacido, con } \\
\text { ingresos totales por debajo de } 3 \text { Salarios Minimos Nacionales. }\end{array}$} \\
\hline 2002 & \\
\hline & \\
\hline 2003 & \\
\hline 2004 & $\begin{array}{l}\text { Hogares contributivos y no contributivos a la seguridad social, con ingresos totales } \\
\text { por debajo de } 3 \text { Salarios Mínimos Nacionales. }\end{array}$ \\
\hline 2005 & \multirow{3}{*}{$\begin{array}{l}\text { Hogares contributivos y no contributivos a la seguridad social, con ingresos totales } \\
\text { por debajo de } 3 \text { Bases de Prestaciones y Contribuciones. }\end{array}$} \\
\hline 2006 & \\
\hline 2007 & \\
\hline 2008 & \multirow{10}{*}{ Índice de Carencias Críticas } \\
\hline 2009 & \\
\hline 2010 & \\
\hline 2011 & \\
\hline 2012 & \\
\hline 2013 & \\
\hline 2014 & \\
\hline 2015 & \\
\hline 2016 & \\
\hline 2017 & \\
\hline
\end{tabular}

Fuente: elaboración propia

La tabla 1 resume el criterio de selección que se utiliza cada año para los diferentes tipos de Asignaciones Familiares en Uruguay. Para generar el pareamiento desde el 2001 hasta el 2007 existe la dificultad de que, estrictamente, las condiciones no definen un algoritmo de selección sino un conjunto de elementos que hacen a la elegibilidad para participar en el programa. Frente a esto se utiliza la condición 
monetaria impuesta por el programa para, de ese modo, estimar un conjunto de determinantes para dichas condiciones y parear en función de los determinantes escogidos con una función de estimación de tipo Mincer (Mincer, 1974). De este modo se replica la estructura de ingresos y de desigualdad de ingresos que en primer lugar llevó a dicho hogar a la condición monetaria. El método que se escoge para generar el algoritmo para la serie 2008-2017 corresponde a un modelo lineal generalizado tipo probit, tal como fue estimada la versión original del ICC (Vigorito et al., 2005).

\section{Análisis}

En primera instancia se propone comenzar el análisis observando el efecto de las Asignaciones Familiares en la asistencia a la Educación Media Básica. Las estimaciones en este caso contemplan a los jóvenes de entre 12 y 17 años y, por tanto, hay jóvenes que poseen algún grado de rezago. No obstante, el efecto edad ya está presente como un elemento que incrementa las probabilidades de experimentar eventos de transición a la adultez públicos y privados.

Gráfico 1. Evolución de la asistencia a Educación Media Básica para jóvenes de 12 a 17 años de los quintiles 1,2 y 3 , pareados de acuerdo al criterio de focalización del programa Asignaciones Familiares, que han sido y no han sido beneficiarios de Asignaciones Familiares en Uruguay. Periodo 2001-2017 excluyendo localidades menores a 5 mil habitantes.

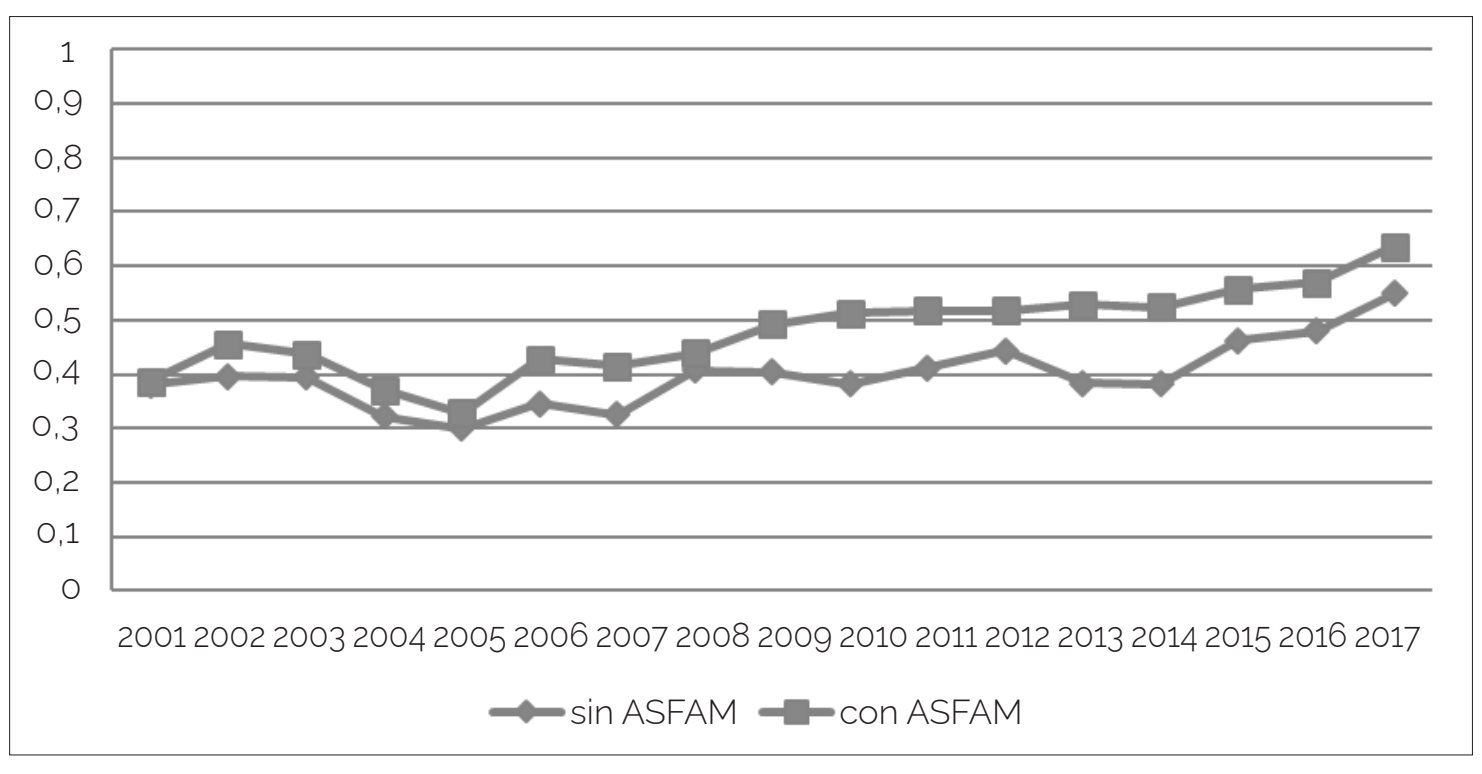

Fuente: elaboración propia en base a ECHs2001-2017. Datos ponderados.

El gráfico 1 representa la evolución de la asistencia a la Educación Media Básica para los jóvenes de entre 12 y 17 años que, perteneciendo a los quintiles 1,2 y 3 , pareados de acuerdo al criterio de asignación de la transferencia, que han sido y no han sido beneficiarios de Asignaciones Familiares en Uruguay. Lo que se observa es que las series evolucionan de manera paralela, principalmente en el recorrido pre tratamiento del 2001 al 2007, para luego ampliar su diferencia en el periodo post-tratamiento. En este caso, observando cada una de las series, se podría esperar algún efecto significativo en el cambio y la ampliación del régimen de Asignaciones Familiares. Más allá de las diferencias en los periodos pre-post, nuevamente se ve una tendencia general de crecimiento de la matrícula durante el periodo. Nuevamente, el supuesto de las tendencias paralelas parece ser modesto. 
Tabla 2. Diferencia en Diferencias (diff in diff) para los jóvenes de entre 12 y 17 años, pertenecientes al quintil 1, 2 y 3, pareados de acuerdo al criterio de focalización de las Asignaciones Familiares, que asisten a la Educación Media Básica, por condición de ser beneficiarios o no de Asignaciones Familiares en Uruguay. Periodo 2001-2017, excluidas las localidades menores a 5 mil habitantes.

\begin{tabular}{|c|c|c|c|c|c|}
\hline & & Asiste EM & Error estándar & $|t|$ & $P>|t|$ \\
\hline \multirow{3}{*}{ Pre } & Control & 0.35 & & & \\
\hline & Tratados & 0.40 & & & \\
\hline & Diff (T-C) & 0.05 & 0.010 & 4.98 & $0.000^{* * *}$ \\
\hline \multirow{3}{*}{ Post } & Control & 0.43 & & & \\
\hline & Tratados & 0.52 & & & \\
\hline & $\operatorname{Diff}(T-C)$ & 0.09 & 0.009 & 10.42 & $0.000^{* * *}$ \\
\hline \multicolumn{2}{|c|}{ Diff-in-Diff } & 0.04 & 0.013 & 2.92 & $0.004^{* * *}$ \\
\hline \multicolumn{6}{|c|}{ Frecuencia } \\
\hline & & pre & post & Total & \multirow{4}{*}{0.02} \\
\hline & htrol & 5164 & 4421 & 9585 & \\
\hline & ados & 7671 & 19226 & 26897 & \\
\hline \multicolumn{2}{|c|}{ Total } & 12835 & 23647 & 36482 & \\
\hline \multicolumn{6}{|c|}{${ }^{* \star *} 99 \%$ n.c.; ${ }^{* *} 95 \%$ n.c. ${ }^{*} 90 \%$ n.ci, . no significativo } \\
\hline \multicolumn{6}{|c|}{ Las medias y los errores estándar de estiman por regresión lineal } \\
\hline
\end{tabular}

Fuente: elaboración propia en base a ECHs 2001-2017. Datos ponderados.

La tabla 2 reporta el impacto de las Asignaciones Familiares en aquellos jóvenes de entre 12 y 17 años que, perteneciendo al quintil 1, 2 y 3, han sido pareados de acuerdo al criterio de asignación de Asignaciones Familiares y que asisten a Educación Media Básica por condición de ser beneficiarios o no de Asignaciones Familiares en Uruguay para el periodo 2001-2017. Lo primero que se observa es que en todos los casos se aprecia un efecto estadisticamente significativo y positivo. El efecto de las Asignaciones Familiares pre tratamiento, antes de la ampliación y reforma del régimen, incrementaba la asistencia a la Educación Media Básica en un $5 \%$, mientras que post tratamiento, el porcentaje de incremento en la asistencia a la Educación Media Básica aumenta hasta alcanzar un 9\%. Estos resultados, tanto en la magnitud como en el sentido y la significación, se aproximan a los reportados en diversos antecedentes, particularmente en Machado et al. (2012), quien reportó un promedio del 11\% para los jóvenes de entre 12 y 17 años de Educación Media Básica (Machado et al., 2012). Con respecto a la diferencia prepost tratamiento, ese $4 \%$ adicional nuevamente resulta significativo y, por tanto, el cambio de régimen favoreció a los jóvenes de los quintiles 1,2 y 3 de entre 12 y 17 años con alta vulnerabilidad que asistian a Educación Media Básica.

Este efecto significativo e incremental reportado en la tabla 2 implica que el cambio en el algoritmo de selección, que focalizó las Asignaciones Familiares en los jóvenes provenientes de los hogares con mayor vulnerabilidad, generó un efecto promedio positivo en relación a aquellos jóvenes igualmente vulnerables que no recibieron la transferencia. Esto es importante, en primer lugar porque cambiando el criterio de focalización e incluyendo población más vulnerable, se lograron mejores resultados. En segundo lugar, al comparar la población prepost, hay un cambio producto de la focalización que, como ya se dijo, introduce más jóvenes de alta vulnerabilidad. No obstante, el cambio en el régimen de 
Asignaciones Familiares del Plan de Equidad funciona. Esto permite pensar en la hipótesis de que el incremento en el monto de la transferencia logra que el subsidio a la demanda educativa funcione.

A continuación resta observar el impacto de las Asignaciones Familiares en la asistencia a la Educación Media Superior. Este ciclo educativo posee algunas peculiaridades respecto a la estimación de un programa de transferencias. En primer lugar, mientras la Educación Media Básica es obligatoria desde la ley de educación de 1971, la Educación Media Superior comienza a serlo desde la ley de educación de 2008. Esto implica que: i) desde 1971 a 2017 son 46 años de reformas y dispositivos de acompañamiento orientados a la universalización de la Educación Media Básica, por lo tanto el perfil de estudiantes de la Educación Media Superior está mucho más seleccionado por diversos procesos familiares, institucionales e individuales que el perfil de estudiante de Educación Media Básica, y: ii) hace 46 años que el límite de la educación formal obligatoria es la Educación Media Básica, por lo tanto el mercado laboral está preparado para absorber la demanda laboral de los individuos con el nivel de habilidades que implica dicho límite en la inversión del capital humano.

En segundo lugar, quienes acceden a la Educación Media Superior tienen, al menos, 14 años de edad, y normativamente terminan el ciclo con un máximo de 18 años. Por lo tanto, incluso asumiendo el conjunto de los estudiantes normativos, los 14 años marcan el inicio de la edad legal para trabajar al tiempo que se comienzan a acumular eventos de transición a la adultez lo que, en los rezagados, fuera de Montevideo y de hogares de bajos ingresos, ocurre en una proporción aún mayor. Un tercer elemento entre quienes asisten a la Educación Media Superior, y de acuerdo al Anuario Estadístico de Educación 2017, es que predomina la matrícula de Secundaria frente a la de UTU en un porcentaje del 72\% contra 27\% (MEC, 2018). De hecho, hasta 1997 no existía la Educación Media Superior técnica de UTU. Por lo tanto, el perfil de los estudiantes de Educación Media Superior ha sido históricamente el de aquellos formados con una orientación preuniversitaria y, por lo tanto, sin habilidades para el mercado laboral que pudieran ser capitalizadas de manera diferencial respecto a la Educación Media Básica.

Estas tres consideraciones respecto a las particularidades de la matrícula de la Educación Media Superior implican una alteración en la función de costos y beneficios, lo que dificulta conocer a priori el efecto de una política de subsidio al costo privado de la demanda educativa. 
Gráfico 2. Evolución de la asistencia a Educación Media Superior para jóvenes de 15 a 17 años de los quintiles 1, 2 y 3, pareados de acuerdo al criterio de focalización del programa Asignaciones Familiares, que han sido y no han sido beneficiarios de Asignaciones Familiares en Uruguay. Periodo 2001-2017 excluyendo localidades menores a 5 mil habitantes.

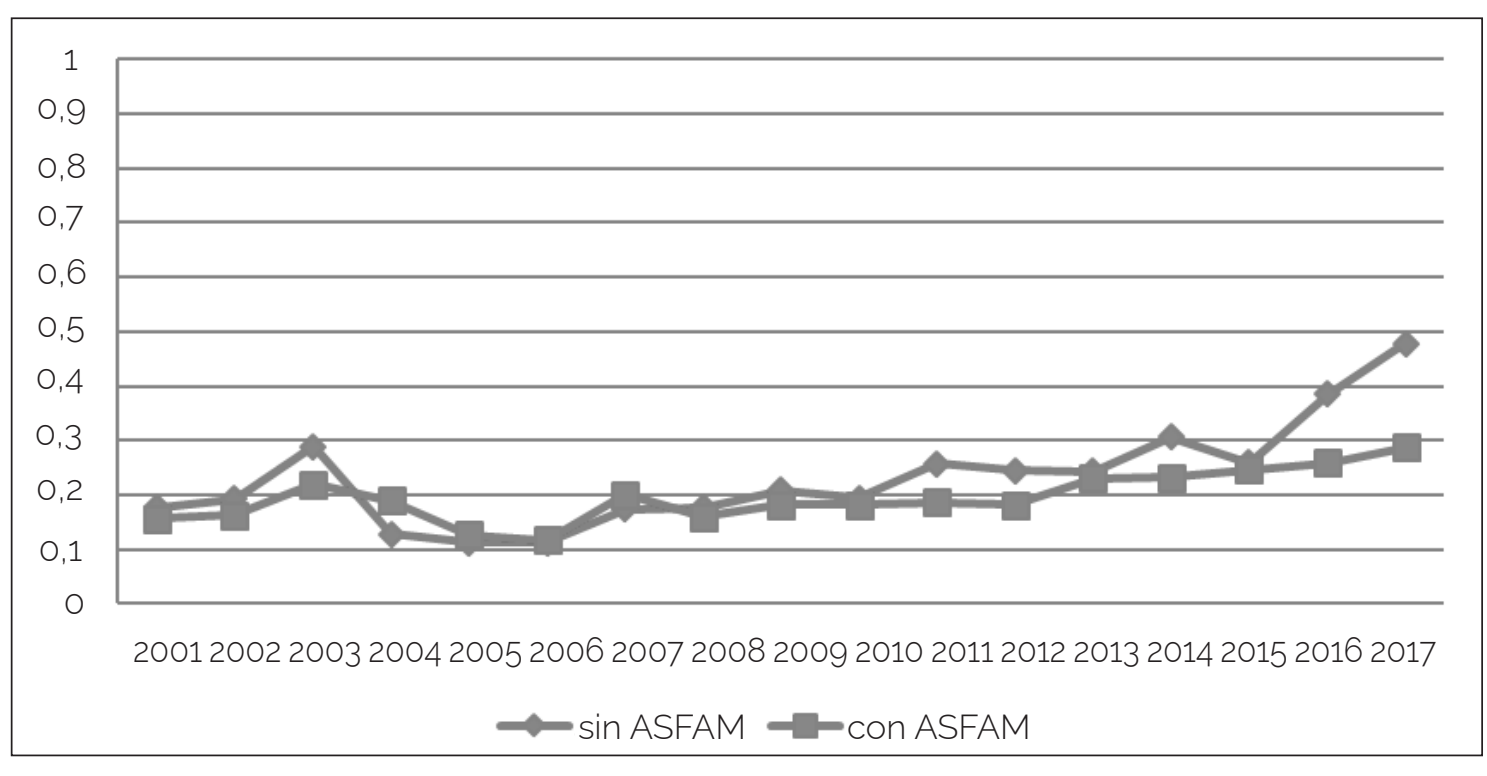

Fuente: elaboración propia en base a ECHs 2001-2017. Datos ponderados.

El gráfico 2 representa la evolución de la asistencia a la Educación Media Superior para los jóvenes de entre 15 y 17 años que, perteneciendo a los quintiles 1, 2 y 3 pareados de acuerdo al criterio de asignación de la transferencia, y que han sido y no han sido beneficiarios de Asignaciones Familiares en Uruguay. Lo primero que se observa es que las series de los beneficiarios y no beneficiarios evolucionan con un margen, entre series, mucho menor que al observar la Educación Media en conjunto o la Educación Media Básica. En segundo lugar, al observar pre-post, a excepción del periodo 2004-2008, predominan en la asistencia a la Educación Media Superior aquellos que no reciben transferencias por concepto de Asignaciones Familiares, tendencia que se acentúa particularmente en 2002-2003 y desde el 2015. Ambos periodos son muy diferentes entre sí pero caracterizados por un descenso significativo en la tasa de empleo, lo que siempre impacta con mayor magnitud en el desempleo juvenil (INE, 2018). En este caso, el supuesto de las tendencias paralelas parece lograr mejor ajuste. 
Tabla 3. Diferencia en Diferencias para los jóvenes de entre 15 y 17 años, pertenecientes al quintil 1 , 2 y 3, pareados de acuerdo al criterio de focalización de las Asignaciones Familiares, que asisten a la Educación Media Superior, por condición de ser beneficiarios o no de Asignaciones Familiares en Uruguay. Periodo 2001-2017, excluidas las localidades menores a 5 mil habitantes.

\begin{tabular}{|c|c|c|c|c|c|}
\hline & & Asiste EM & Error estándar & $|t|$ & $P>|t|$ \\
\hline \multirow{3}{*}{ pre } & Control & 0.17 & & & \\
\hline & Tratados & 0.17 & & & \\
\hline & $\operatorname{Diff}(T-C)$ & 0.00 & 0.012 & -0.25 & 0.802 \\
\hline \multirow{3}{*}{ post } & Control & 0.29 & & & \\
\hline & Tratados & 0.21 & & & \\
\hline & Diff $(T-C)$ & -0.07 & 0.010 & 7.23 & $0.000^{* * *}$ \\
\hline \multicolumn{2}{|c|}{ Diff-in-Diff } & -0.07 & 0.015 & 4.58 & $0.000^{* * *}$ \\
\hline \multicolumn{6}{|c|}{ Frecuencia } \\
\hline & & pre & post & Total & \multirow{4}{*}{0.01} \\
\hline & rol & 2756 & 2641 & 5397 & \\
\hline & dos & 3498 & 9217 & 12715 & \\
\hline & & 6254 & 11858 & 18112 & \\
\hline \multicolumn{6}{|c|}{${ }^{\star \star *} 99 \%$ n.c; ${ }^{* \star} 95 \%$ n.c; ${ }^{*}$ 90\% n.ci, . no significativo } \\
\hline \multicolumn{6}{|c|}{ Las medias y los errores estándar de estiman por regresión lineal. } \\
\hline
\end{tabular}

Fuente: elaboración propia en base a ECHs 2001-2017. Datos ponderados.

La tabla 3 reporta el impacto de las Asignaciones Familiares en aquellos jóvenes de entre 15 y 17 años que, perteneciendo al quintil 1, 2 y 3, han sido pareados de acuerdo al criterio de asignación de Asignaciones Familiares, que asisten a Educación Media Superior por condición de ser beneficiarios o no de Asignaciones Familiares en Uruguay para el periodo 2001-2017. Existen tres elementos a tener en cuenta en esta tabla. En primer lugar, las Asignaciones Familiares no tienen un efecto significativo pre-tratamiento y, por tanto, la asistencia a la Educación Media Superior entre beneficiarios y no beneficiarios es equiprobable.

Asimismo, el efecto anteriormente observado resulta curioso debido a que un programa de transferencias no solo no funciona, sino que lo hace en el sentido opuesto al esperado. Una hipótesis plausible involucra al mercado de empleo, y el hecho de que frente al desempleo los individuos se vuelcan al sistema educativo. En tercer lugar, la Educación Media Superior posee bajas tasas de asistencia, por lo tanto un efecto derrame del mercado laboral al sistema educativo es rápidamente detectado por las tasas de asistencia. Esto les ocurre a todos, con excepción de aquellos que poseen un subsidio con una condicionalidad flexible, que los hace menos sensibles a las variaciones de las tasas de empleo y, por lo tanto, el desempleo no los retorna al sistema educativo. Durante la crisis del año 2002 esta tendencia parece no haberse acentuado, posiblemente porque el monto de las transferencias en ese entonces era sensiblemente menor, no actuando como un amortiguador de los efectos del desempleo. 


\section{Conclusiones}

Con respecto a la primera hipótesis, que sostiene que el subsidio a la demanda educativa genera efectos positivos y estadisticamente significativos en la persistencia en la Educación Media Básica, se supone que esto ocurre en virtud de que el subsidio logra cubrir el costo educativo de continuar estudiando, y que la utilidad dentro de los primeros años de Educación Media es baja. Esta hipótesis no ha sido falseada. De hecho se han encontrado efectos positivos y estadisticamente significativos del subsidio a la demanda educativa en la persistencia en la Educación Media Básica.

Sin embargo, la segunda hipótesis donde el subsidio a la demanda educativa genera efectos positivos y estadísticamente significativos en la permanencia en la Educación Media Superior sí se falsea. Si el argumento de que el subsidio logra cubrir los costos educativos es correcto para la Educación Media Básica pero no para la Educación Media Superior, puede querer sugerir -entre otras cosas- que los costos de la Educación Media Superior son superiores a los de la Educación Media Básica.

Las consecuencias de la contrastación de las hipótesis 1 y 2 tienen una doble implicancia. Por una parte, el monto de la transferencia logra subsidiar el gasto educativo en Educación Media Básica por medio de cuatro elementos: i) logra cubrir de manera significativa los costos directos de la escolarización; ii) el costo indirecto no es suficientemente elevado en virtud de que la utilidad educativa de educación primaria parece ser significativamente menor a la utilidad educativa de acreditar Educación Media Básica; iii) la utilidad educativa de acreditar Educación Media Básica implica una inversión atractiva y razonable para los hogares de menores ingresos, y; iv) en términos de ciclo vital, el efecto edad aún no comenzó a actuar como catalizador del inicio de otras trayectorias laborales y familiares asociadas al final de la moratoria vital. En el caso de la Educación Media Superior, el subsidio al costo directo es muy modesto y, además, los costos indirectos son muy altos y la utilidad educativa es muy baja.

Por otra parte, las políticas de transferencia actúan en un mapa de mucha desigualdad asociada a otros elementos, y por tanto su rol es modesto ya que incluso si fuera 100\% efectivo como política de subsidio, la brecha que se abre durante el tránsito entre ciclos, y que no está asociada a recursos, aún persiste y actúa como un poderoso sistema de filtro en la matrícula entre Educación Media Básica y Educación Media Superior.

Se podría esperar que lo mismo sucediera con otros programas de diferente diseño con componentes de transferencias condicionadas, que apuntan a una vulnerabilidad económica que no necesariamente se asocia con la vulnerabilidad educativa. Como se ha reseñado en los antecedentes, muchos de estos programas tienen efectos significativos en la asistencia y no en la acreditación del grado o culminación del ciclo. Si bien en buena medida esto se asocia al diseño del programa y al mecanismo de renovación de la transferencia, también existe un componente asociado a la diferencia entre asistir y acreditar. El tiempo necesario para culminar la Educación Media normativamente es de 6 años, pero varía de acuerdo a la evolución de las trayectorias de los estudiantes en la medida que simplemente crecen. De este modo existe un conjunto sistemático de individuos que incluso si la utilidad educativa de culminar la Educación Media Superior fuera significativa, implicaria una inversión a tan largo plazo, superior a 6 años, que terminaría no valiendo la pena. Por lo tanto, una herramienta especíica como la repetición, diseñada para apuntalar los conocimientos no adquiridos durante el grado lectivo, funciona en contraposición a todas las políticas de transferencias, que se extinguen a los 18 años. Es decir, y como ya se ha señalado, incluso si las 
transferencias funcionaran, a los 18 años los jóvenes ven desaparecer el único elemento que ha contribuido a su escolarización debido a que han repetido alguna vez. Esta situación se convierte en una paradoja cuando se considera que vulnerabilidad, rezago y transferencias parecen elementos tautológicos, donde las razones por las que se le otorga el beneficio a un individuo en primer lugar, son las mismas razones por las cuales se le quita dicho beneficio posteriormente.

Notas:

Aprobación final del artículo: editora responsable Mag. Verónica Zorrilla de San Martín.

Contribución de autoria: la elaboración del artículo es obra única del autor. 


\section{Bibliografía}

ABADIE, A., \& IMBENS, G. (2006). Large sample properties of matching estimators for average treatment effects [Propiedades de las grandes muestras en la estimación del emparejamiento de los efectos de tratamiento promedio]. Econometrica, 74(1), 235-267. https://doi.org/10.1111/j.1468-0262.2006.00655.x

AMARANTE, V., ARIM, R., DE MELO, G., \& VIGORITO, A. (2009). Transferencias de ingresos y asistencia escolar. Una evaluación ex-ante de esquemas alternativos en Uruguay (Serie Documentos de Trabajo / FCEA-IE; DT06/Og). https://hdl. handle.net/20.500.12008/4183

BECKER, G. (1993). Human capital: a theoretical and empirical analysis, with special reference to education [El capital humano: análisis teórico y empírico referido

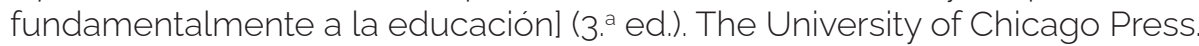

BÉRGOLO, M., DEAN, A., PERAZZO, I., \& VIGORITO, A. (2015). Evaluación impacto del programa Asignaciones Familiares-Plan de Equidad y la Tarjeta Alimentaria del MIDES. Evaluación cuantitativa del impacto de Asignaciones Familiares-Plan de Equidad. http://dspace.mides.gub.uy:8080/xmlui/handle/123456789/1073

BUCHELI, M., CABELLA, W., \& VIGORITO, A. (2005). Asignaciones familiares, pensiones alimenticias y bienestar de la infancia en Uruguay. http://dspace. mides.gub.uy:8080/xmlui/handle/123456789/237

CAMPBELL, D., \& COOK, T. (1979). Quasi-experimentation: Design and analysis for field settings [Cuasi-experimentación: diseño y análisis para trabajos de campo]. Houghton-Mifflin.

CONLEY, T. G., \& TABER, C. R. (2011). Inference with "difference in differences" with a small number of policy changes [Inferencia con "diferencia en diferencias" con una pequeña cantidad de cambios de polítical. Review of Economics and Statistics, 93(1), 113-125. https://doi.org/10.1162/REST_a_00049

DA SILVEIRA, P. (2009). Padres, Maestros y Políticos. Taurus.

DINEM-MIDES. (2014). ¿Qué es el índice de carencias críticas? Montevideo.

DINEM-MIDES. (2015). Análisis y perspectivas para los programas de transferencias: Asignaciones Familiares y Tarjeta Uruguay Social. http://dspace.mides.gub. uy:8080/xmlui/handle/123456789/1045

DONALD, S. G., \& LANG, K. (2007). Inference with Difference-in-Differences and Other Panel Data [Inferencia con Diferencia en Diferencias y otros datos panel]. Review of Economics and Statistics, 89(2), 221-233. https://doi.org/10.1162/ rest.89.2.221

IMBENS, G. W., \& WOOLDRIDGE, J. M. (2009). Recent Developments in the Econometrics of Program Evaluation [Desarrollos recientes en la econometría de la evaluación de programas]. Journal of Economic Literature, 47(1), 5-86. https://doi.org/10.1257/jel.47.1.5

INE. (2018). Anuario Estadistico Nacional 2018. HTTPS://WWW.INE.GUB.UY/ DOCUMENTS/10181/559909/ANUARIO+ESTADÍSTICO+NACIONAL+2018/466 60CE3-EB26-484E-B295-F4327499DE8B 
MACHADO, A., CASTAINGS, M., CHIOSSI, J., DIBOT, G., GONZÁLEZ, M., MORENO, M., ROJO, V., RYAN, M., \& TERRA, F. (2012). El nuevo régimen de Asignaciones Familiares. Su impacto en la participación y permanencia en el Ciclo Básico de Educación Media. http://dspace.mides.gub.uy:8080/xmlui/ handle/123456789/1246

MEC. (2018). Anuario Estadistico de Educación 2017. https://icau.mec.gub.uy/ innovaportal/file/11078/1/anuario-2017.xlsm

MIDAGLIA, C. (2009). Entre la tradición, la modernización ingenua y los intentos de refundar la casa: la reforma social en el Uruguay de las últimas tres décadas. En C. Barba Solano (Ed.), Retos para la integración social de los pobres en América Latina (pp. 85-107). CLACSO. http://biblioteca.clacso.edu.ar/clacso/ gt/20160223033921/ogmidaglia.pdf

MINCER, J. (1974). Schooling, Experience and Earnings IEscolarización, experiencia y gananciasl. National Bureau of Economic Research. https://www.nber.org/ books-and-chapters/schooling-experience-and-earnings

ROSSEL, C., COURTOISIE, D., \& MARSIGLIA, M. (2014). Programas de transferencias, condicionalidades y derechos de la infancia. Apuntes a partir del caso del Uruguay. https://repositorio.cepal.org/bitstream/handle/11362/37328/1/S1420825_ es.pdf

SEN, A. (2000). Desarrollo y libertad. Planeta.

SHADISH, W., COOK, T., \& CAMPBELL, D. (2002). Experimental and QuasiExperimental Designs for Generalized Causal Inference [Diseños experimentales y quasi-experimentales para Inferencia Causal Generalizada]. Houghton-Mifflin.

VIGORITO, A., ARIM, R., \& AMARANTE, V. (2005). Metodología para la selección de los participantes en el plan de emergencia social. http://guiaderecursos. mides.gub.uy/innovaportal/file/6570/1/metodologia-de-la-seleccion-de-lapoblacion-del-plan-de-atencion-nacional-a-la-emergencia-social-panes.pdf

VIGORITO, A., \& COLAFRANCESCHI, M. (2013). Uruguay: evaluación de las políticas de transferencias. La estrategia de inclusión y sus desafios. En R. Rofman (Ed.), Hacia un Uruguay más equitativo. Los desafios del sistema de protección social (pp. 73-142). Banco Mundial. https://www.worldbank.org/content/dam/ Worldbank/document/LAC/hacia_un_uruguay_mas_equitativo_web.pdf

WOODHALL, M. (2004). Cost-benefit analysis in educational planning IAnálisis de

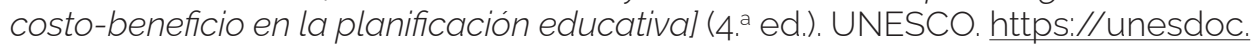
unesco.org/ark:/48223/pfo000139042 